\title{
eJRIEPS
}

Ejournal de la recherche sur l'intervention en éducation physique et sport

32 | 2014

Varia

\section{Perceptions d'élèves du secondaire concernant leurs inducteurs émotionnels en EPS}

Oriane Petiot, Jean-François Desbiens et Jérôme Visioli

\section{OpenEdition}

Journals

Édition électronique

URL : http://journals.openedition.org/ejrieps/2008

DOI : 10.4000/ejrieps.2008

ISSN : 2105-0821

Éditeur

ELLIADD

\section{Référence électronique}

Oriane Petiot, Jean-François Desbiens et Jérôme Visioli, « Perceptions d'élèves du secondaire concernant leurs inducteurs émotionnels en EPS », eJRIEPS [En ligne], 32 | 2014, mis en ligne le 01 avril 2014, consulté le 03 octobre 2019. URL : http://journals.openedition.org/ejrieps/2008 ; DOI : 10.4000/ejrieps.2008

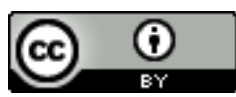

La revue eJRIEPS est mise à disposition selon les termes de la Creative Commons Attribution 4.0 International License. 
eJRIEPS 32 avril 2014

Perceptions d'élèves du secondaire concernant leurs inducteurs émotionnels en EPS

Oriane Petiot ${ }^{\star}$, Jean-François Desbiens** \& Jérôme Visioli**

* UFR STAPS de l'université Rennes 2 et ENS de Rennes, FRANCE

${ }^{* *}$ CRIFPE, Faculté d'éducation, Université de Sherbrooke, QUEBEC, CANADA

*** UFR STAPS de l'Université Rennes 2, FRANCE

Résumé

L'objectif de cet article est d'identifier, de décrire et de hiérarchiser les inducteurs des émotions positives et négatives ressenties par des élèves du secondaire en EPS. Les perceptions des élèves ont été recueillies à l'aide d'un questionnaire basé sur la méthode des incidents critiques (Flanagan, 1954) leur demandant de raconter un épisode marquant qu'ils ont vécu en EPS depuis le début de l'année scolaire. L'analyse inductive des 369 épisodes recueillis a fait émerger une taxonomie de huit inducteurs émotionnels. L'inducteur regroupant le plus d'incidents critiques fait référence à l'enseignant d'EPS, notamment à ses comportements interpersonnels à l'égard des élèves. Les objectifs, le contenu et la mise en œuvre des séances sont également discutés au regard de leurs liens avec les émotions vécues en EPS.

Mots clés: émotions, perceptions sociales, incidents critiques, comportements interpersonnels de l'enseignant

\section{Introduction}

Les travaux scientifiques traitant des émotions ont connu un essor considérable depuis une quinzaine d'années, que ce soit dans une perspective neurobiologique (Belzung, 2007), cognitiviste (Scherer, Schorr \& Johnstone, 2001), psychologique et culturelle (Clot, 1999), éthologique (Cyrulnik, 2006), anthropologique et sociologique (Le Breton, 1998) ou encore philosophique (Dumouchel, 1999). Pour leur part, les chercheurs en sciences de l'éducation incluant ceux des STAPS, ont participé à ces nouveaux débats, notamment sous l'angle des émotions ressenties par les enseignants d'EPS (Ria, 2005 ; Visioli, 2009 ; voir Visioli, Petiot \& Ria, soumis, pour une revue). D'un point de vue plus professionnel, 


\section{eJRIEPS 32 avril 2014}

certains se sont penchés spécifiquement sur la question des émotions ressenties par les élèves en cours d’EPS (Gagnaire \& Lavie, 2007 ; Haye, 2011).

Dans un rapport intitulé « Image du sport scolaire et pratiques d'enseignement au collège et au lycée 2005-2006 », publié en 2007 sur le site du Ministère de l'éducation nationale, les élèves, garçons comme filles, ont exprimé une représentation très positive des cours d'EPS. Plus précisément, lorsqu'il leur a été demandé ce que les cours d'EPS leur apportaient, les items qu'ils ont cité en premier se situaient dans le champ du plaisir et de l'épanouissement : 68\% d'entre eux ont déclaré prendre du plaisir à pratiquer des activités physiques et sportives. Toutefois, une enquête réalisée quelques années plus tôt par la Direction Pédagogique Régionale de Lille (Lefort, 2001) a révélé que l'EPS a reculé dans le classement des disciplines préférées des élèves, notamment en ce qui concerne les filles lycéennes. Deslaurier (2007) a d'ailleurs montré empiriquement que les filles, au fur et à mesure de leur scolarité, semblent exprimer un avis particulièrement critique vis-à-vis de l'EPS. Ainsi, la relation entre plaisir des élèves et pratique des APSA mérite d'être examinée avec attention afin de mieux comprendre de quelle manière elle se construit aux plans didactique et pédagogique (Delignières \& Garsault, 2004), à l'heure où les législateurs français dotent l'EPS de finalités ambitieuses touchant notamment la gestion par l'élève de sa vie physique actuelle et future (Bulletin officiel spécial nø du 28 août 2008). Delignières et Garsault (2004) sont allés jusqu'à affirmer que le plaisir de pratiquer des activités physiques, sportives et artistiques (APSA) doit être considéré comme une acquisition fondamentale en EPS, tant elle conditionne les autres acquisitions attendues, leur réinvestissement et leur utilité. En prenant comme support de recherche les pratiques physiques d'entretien, Perrin (1993) a d'ailleurs montré que les adultes adoptant une pratique physique régulière sont bien davantage guidés par le plaisir procuré par l'exercice que par des motivations hygiénistes. II est même ressorti que les individus qui invoquaient l'exercice corporel comme une nécessité pour la santé n'en faisaient pas ou peu comme s'il ne s'agissait pour eux que d'adopter un point de vue socialement désirable.

Dans une perspective sociocognitive, Lazarus (1991) a insisté sur l'étroitesse des liens entre l'émotion, la cognition et l'environnement. Selon lui, les interactions entre l'environnement et un individu sont susceptibles d'affecter son bien-être, et comporteraient une signification émotionnelle potentielle. Les émotions induites par ces interactions déboucheraient alors sur une ou plusieurs actions de l'individu destinées à rétablir et/ou maintenir des conditions optimales de bien-être, réaffirmant les relations étroites entre émotion et engagement. Les émotions existeraient pour nous aider à faire face aux défis, 


\section{eJRIEPS 32 avril 2014}

aux stress et aux problèmes de la vie (Reeve, 2009). Ce sont alors les inducteurs, c'est-àdire les éléments qui déclenchent les composantes émotionnelles, qui amèneraient à une adaptation de l'individu pour faire face à la situation. Ceci limite la survenue d'émotions négatives et favoriser la survenue d'émotions positives (Lazarus, 1984). Malgré des divergences théoriques, Nugier (2009) mais aussi Sander et Scherer (2009) ont estimé que la plupart des chercheurs s'accordaient à définir les émotions comme un phénomène multicomponentiel, c'est-à-dire composé de plusieurs éléments interdépendants : (a) des comportements expressifs; (b) des évaluations cognitives; (c) des réactions physiologiques; (d) des tendances à l'action; (e) l'expérience subjective (ou sentiments subjectifs), c'est à dire ce qu'on pense ou dit ressentir. Cette dernière composante suggère la plus-value de s'intéresser aux perceptions singulières pour déterminer le vécu émotionnel des acteurs. Ce vécu émotionnel investigué peut être celui d'élèves en EPS.

L'objectif de cette étude est d'approfondir cette question du plaisir en EPS, qui reste particulièrement controversée, mais aussi de l'élargir à l'ensemble des émotions ressenties par les élèves en EPS. Nous souhaitons plus particulièrement mettre la focale sur les inducteurs émotionnels dans le but de pondérer l'importance de ces inducteurs à l'aide d'une approche partant du point de vue intrinsèque des élèves. En d'autres termes, nous proposons dans un premier temps d'identifier de manière inductive les inducteurs qui déclenchent des émotions positives et négatives ressenties en EPS par les élèves. Dans un second temps, nous chercherons à hiérarchiser ces inducteurs émotionnels sur la base de leur fréquence d'apparition dans les propos des élèves.

La documentation consultée, et empreinte à des champs scientifiques variés, permet déjà d'anticiper certains inducteurs susceptibles de générer des émotions chez les élèves. Pour l'essentiel, ces facteurs touchent de près les notions étroitement reliées de climat d'apprentissage (Martel, Brunelle \& Spallanzani, 1991) et de climat motivationnel (Watson \& Clocksin, 2013). Le climat d'apprentissage est caractérisé par la qualité et la quantité de l'engagement des élèves. Il est déterminé par la nature des interactions entre l'enseignant et ses élèves de même qu'entre les élèves, la nature des activités proposées et la façon dont est défini le contexte d'apprentissage. Pour sa part, le climat motivationnel est défini par l'interprétation que se sont forgés les élèves sur la base d'indices émis par l'enseignant au travers de ses pratiques d'évaluation et de responsabilisation formelles et informelles. Plus particulièrement, le climat motivationnel varie selon qu'il est orienté vers la maitrise ou vers la compétition. Les caractéristiques d'un climat de maitrise sont par exemple le choix de tâches selon le niveau d'habileté de l'élève, la reconnaissance des 
eJRIEPS 32 avril 2014

efforts et des progrès des élèves et un rapport positif à l'erreur, ou encore une certaine flexibilité et une hétérogénéité des formes de regroupements. Les effets d'un tel climat sur l'activité de l'élève ont trait à la mise en place de stratégies d'études plus efficaces et plus approfondies, une attitude positive face au cours et la conviction que l'effort mène à la réussite, sans que de meilleures notes soient pour autant nécessairement obtenues. Le climat de compétition suggère quant à lui plutôt que l'enseignant contrôle davantage les décisions liées à l'apprentissage et/ou à l'organisation du cours, qu'il encourage et valorise la performance ou encore qu'il adopte une évaluation publique et plus normée et forme des groupes de niveaux explicitement organisés. Un tel climat semble avoir pour effet de mettre la focale sur la comparaison sociale, à privilégier une attribution de l'échec à un déficit de compétence et à induire davantage d'anxiété, de tricherie, ainsi qu'une diminution de l'effort et de la persévérance (Sarrazin, Tessier et Trouilloud, 2006).

Plusieurs chercheurs ont déclaré que l'enseignant d'EPS possédait un statut privilégié pour influencer l'ambiance qui règne en classe (Siedentop, 1994, pour une revue). Dans une perspective de type processus-produit, Cheffers et Mancini (1978) affirmaient, il y a plus de trente ans, que si nous observons et comptabilisons les feed-back émis par l'enseignant à ses élèves, celui-ci n'utilise pratiquement pas de comportements comme l'écoute active, l'acceptation des sentiments et des idées des élèves. Ils ne les félicitent pas souvent et les questionnent peu. De plus, les comportements qui démontrent de la sympathie et de l'empathie sont presque inexistants. Quelques années plus tard, McLeish (1981), qui a adopté le même type de méthodologie, a corroboré ces propos en affirmant que le renforcement positif, qui constitue pourtant un principe d'apprentissage majeur, n'est que peu usité par les enseignants. Plus récemment, d'autres chercheurs ont montré dans une acception cognitiviste que si nous nous intéressons à ce que perçoivent les élèves en classe, l'enseignant reste déterminant dans leur opinion de la discipline et, plus généralement, de l'école. Dans une étude visant à déterminer les raisons du décrochage scolaire, Savoie-Zajc (1994) a par exemple interviewé quinze élèves québécois qui songeaient à abandonner leurs études. Les entretiens réalisés ont attesté du caractère central de la relation enseignant - élève dans l'appréciation et la critique que les élèves font de l'école. D'autres travaux (Galand \& Phillipot, 2005; Pekrun \& Stephens, 2012) ont établi que des relations positives entre enseignants et élèves réduisent la colère et l'ennui et renforcent l'intérêt des apprenants, mais aussi que les manifestations de plaisir et d'enthousiasme par l'enseignant sont reliées positivement au plaisir déclaré par les élèves, suggérant une transmission positive des émotions du maitre vers les apprenants. 


\section{eJRIEPS 32 avril 2014}

Considérés dans leur ensemble, ces éléments appuient l'idée soutenue par Felouzis (1997) selon laquelle la relation éducative est fortement marquée par l'affectivité.

Les camarades de classe jouent également un rôle majeur dans la construction de l'élève, notamment durant l'adolescence (Braconnier, 1998). À cet âge, la vie en groupe, les rencontres, le faire ensemble, ont une valeur primordiale (Parlebas, 2005). Dans le modèle TPSR (Teaching Personal and Social Responsibility) développé et promu en EPS par Don Hellisson, par exemple, le respect ainsi que la bienveillance et l'aide apportée par les élèves les uns envers les autres constituent des niveaux de responsabilité inscrits formellement dans une démarche développementale de l'intervention (Watson \& Clocksin, 2013). II faut ajouter à l'environnement humain constitué de l'enseignant et des pairs la nature des APSA proposées aux élèves. Au plan anthropologique, Jeu (1977) a proposé une classification des APSA selon leur signification émotionnelle, reprise par Gagnaire et Lavie (2005) avec un regard plus professionnel, selon les modalités didactiques des APSA. La pratique d'une APSA s'accompagne d'émotions particulières, variables d'un individu à l'autre et selon sa nature. Famose (2001) maintient en outre que ce sont la pérennisation de l'estime de soi, son développement et sa protection qui peuvent le plus fortement influencer le comportement motivationnel de l'élève en EPS. Chaque réussite ou chaque échec de l'élève dans la pratique des APSA peut donc influencer ses émotions ressenties en EPS. En clair, si elle exerce indéniablement une influence sur le climat d'apprentissage (Martel et al., 1991), la programmation des APSA et leur traitement didactique par l'enseignant influe vraisemblablement aussi sur les émotions ressenties par les élèves durant les cours.

Plusieurs chercheurs se sont d'ailleurs penchés sur la prise en compte des aspects émotionnels par les enseignants d'EPS. Dans une perspective située, Pérez (1999) a étudié l'activité d'une enseignante lors d'une leçon de gymnastique auprès d'élèves de $5^{\text {ème }}$. L'enseignante cherchait à exploiter le ressenti émotionnel des élèves, notamment lié à la prise de risque, alternant incitations amicales voire provocatrices, mais aussi des paroles visant à rassurer les élèves. L'enseignante faisait donc en sorte de capter les émotions des élèves pour réagir de façon appropriée dans le but de favoriser leur engagement et de les inciter à passer devant les autres. Plus encore, dans un travail doctoral mené auprès de huit enseignants d'EPS experts, Visioli (2009) a montré que les enseignants optent pour des comportements empathiques en vue d'atteindre des objectifs d'apprentissage. Conformément à ces études, nous considérons que la réalité d'une situation importe moins que l'interprétation cognitive de cette situation par les personnes 


\section{eJRIEPS 32 avril 2014}

interrogées (Adler \& Towne, 1998) de sorte qu'il nous semble primordial d'accorder le primat au point de vue des acteurs. Les élèves, qui ont expérimenté une multitude d'environnements d'apprentissage depuis le début de leur scolarité (Fraser, 2001), semblent d'ailleurs capables de rapporter ce qu'ils perçoivent dans le processus d'apprentissage (Weinstein, 1985). Toutefois, ces recherches menées dans une perspective située ont été centrées sur le cours d'action de l'enseignant et n'ont pas eu pour objet d'étudier les perceptions des élèves sur l'action en EPS. Delignières et Pérez (1998) ont, quant à eux, élaboré un questionnaire mesurant le plaisir des élèves en EPS, en mettant l'accent sur la dimension subjective du plaisir ressenti. Si cette approche permet assurément de recueillir les perceptions des élèves, reste que ce questionnaire obéit à une démarche déductive limitant la possibilité pour les acteurs de faire état de l'ensemble de leurs états affectifs ressentis et susceptibles d'influencer leurs comportements en classe.

Ce choix d'entrer par les perceptions exprimées des élèves suggère finalement d'envisager la terminologie des émotions dans un sens large, c'est-à-dire d'inclure les états affectifs s'apparentant davantage aux sentiments ou encore aux humeurs, particulièrement difficiles à différencier sans équivoque (Damasio, 2003 ; Reeve, 2009). Alors que les émotions constituent des moments saillants, ponctuels et nettement isolables du flux continuel des états affectifs, les sentiments sont de l'ordre de l'expérience des émotions. La joie, par exemple, peut aussi bien être entendue comme une émotion fondamentale (Ekman, 1992), ressentie de manière intense et soudaine, que comme un sentiment ou une humeur plus durable et d'intensité modérée (Frijda, 1996). Nous partirons donc des déclarations des participants en distinguant seulement leurs émotions à partir de leur caractère positif ou plaisant ou encore négatif ou déplaisant (Pekrun \& Stephens, 2012). Nous chercherons à positionner sur ce continuum l'ensemble des émotions rapportées par les élèves et susceptibles de générer des conduites particulières en EPS.

\section{Méthodologie}

\subsection{Sélection des participants}

L'étude a été menée auprès d'un échantillon non probabiliste composé de 369 élèves (227 filles et 142 garçons) du secondaire, scolarisés au sein d'une cité scolaire française située en milieu urbain. Ils étaient répartis en 16 classes allant de la $5^{\text {ème }}$ à la $1^{\text {ère }}$ sous la responsabilité de huit enseignants d'éducation physique. Chacun de ces huit enseignants 


\section{eJRIEPS 32 avril 2014}

(4 hommes et 4 femmes) de l'établissement (Tableau 1) était responsable de deux classes. Le chef d'établissement puis les enseignants d'EPS ont été contactés et rencontrés pour leur présenter le projet de recherche. La participation des élèves et des enseignants a été obtenue sur une base volontaire après une présentation du projet à chacune des classes. Un formulaire de consentement éclairé a alors été signé par les parents des élèves et la direction d'établissement. Les enseignants ont également signé un formulaire semblable.

Tableau I. Répartition des 16 classes participant à l'étude en fonction les huit enseignants d'EPS de l'établissement.

Notes. 1ère L : première option littéraire; 1ère ES : première option économique et social; 1ère $S$ : première option scientifique. Les dénominations utilisées sont des pseudonymes.

\begin{tabular}{lllll}
\hline Enseignants & Âge & Expérience & Classe 1 (C1) & Classe 2 (C2) \\
\hline Alisée & 25 & 1 & $5^{\text {eme }}$ & $5^{\text {eme }}$ \\
Léa & 32 & 6 & $4^{\text {eme }}$ & $3^{\text {ème }}$ \\
Olivier & 40 & 16 & $5^{\text {ème }}$ & $3^{\text {ème }}$ \\
Jérôme & 51 & 8 & $4^{\text {eme }}$ & $3^{\text {ème }}$ \\
Jean & 48 & 24 & $2^{\text {nd }}$ & $2^{\text {nd }}$ \\
Stéphane & 52 & 30 & $2^{\text {nd }}$ & $2^{\text {nd }}$ \\
Jocelyne & 34 & 12 & $1^{\text {ere }} \mathrm{ES}$ & $1^{\text {ere }} \mathrm{S}$ \\
Justine & 43 & 19 & $1^{\text {ere }} \mathrm{L}$ & $1^{\text {ere }} \mathrm{S}$ \\
\hline \multirow{5}{*}{} & $\mathrm{M}=40,6$ & $\mathrm{M}=14,5$ & Total $=8$ & Total $=8$ \\
& Étendue $=27$ & Étendue $=29$ & & \\
& Écart-type $=$ & Écart-type $=$ & & \\
& 9,1 & 9,1 & &
\end{tabular}

\subsection{Collecte des données}

2.2.1. Instruments de collecte de données.

Les données ont été collectées à l'aide d'une fiche documentaire. La première partie de cette fiche consistait en un questionnaire sociodémographique. Celui-ci a été distribué aux enseignants et aux élèves afin de recueillir des données sur leur âge, leur sexe ainsi que, dans le cas des seconds, le nombre d'années d'expérience en enseignement de l'EPS. La seconde partie de la fiche documentaire distribuée exclusivement aux élèves concerne spécifiquement un indicent critique (Flanagan, 1954; Brunelle, Drouin, Godbout \& Tousignant, 1988 ; Preskill, 1996) de leur choix dont ils sont invités à rendre compte. L'incident critique est, depuis une vingtaine d'années, une technique utilisée par des chercheurs en activité physique (Marzouk \& Tousignant, 1992 ; Gagnon, Martel, Brunelle, Tousignant \& Spallanzani, 2006) pour obtenir un portrait détaillé d'une situation 


\section{eJRIEPS 32 avril 2014}

d'enseignement en fonction de leur objet de recherche. Comme le montre l'exemple cidessous, il s'est agi d'amener les élèves à relater un évènement marquant vécu en EPS depuis le début de l'année scolaire.

Fiche de description de l'incident critique distribuée aux élèves :

Je te demande de me raconter avec le plus de détails possible un évènement marquant qui s'est déroulé durant un de tes cours d'EPS avec cet enseignant depuis le début de l'année scolaire (mois de septembre).

Tu as le choix entre deux types d'évènements :

- Un évènement durant lequel tu as ressenti des émotions très positives;

- Un évènement durant lequel tu as ressenti des émotions très négatives.

II faut donc que cet évènement ait fait naitre chez toi beaucoup d'émotions !

Tu dois me raconter un seul évènement! Si tu en as plusieurs en tête, choisis celui qui a été le plus fort, qui t'a fait ressentir le plus d'émotions !

Suite à cette consigne, les élèves ont eu à cocher la valence de l'évènement qu'ils ont choisi de raconter.

Coche la bonne case :

Je vais raconter un évènement durant lequel j'ai ressenti des émotions très positives.

Je vais raconter un évènement durant lequel j'ai ressenti des émotions très négatives.

De manière à guider l'élève dans son récit et à l'inciter à nommer précisément les émotions qu'il a ressenties, nous avons inclus deux questions à la suite de cette consigne et précisé le nombre de lignes attendues afin d'obtenir un maximum de détails par les élèves.

1) Parle-moi de l'évènement que tu as choisi : que faisais-tu ? Que s'est-il passé ? Apporte autant de détails que tu le peux. Écris au moins 5 lignes.

2) Parle-moi des émotions que tu as ressenties précisément ! Écris au moins 3 lignes.

2.2.2. Procédure de collecte des données

La collecte des données s'est tenue en janvier et en février 2012 à des moments convenus avec les enseignants d'EPS et la direction de l'établissement. Les élèves ont été rencontrés pendant une période d'une heure prise sur le temps du cours d'EPS. II a été convenu au préalable avec chaque enseignant qu'ils seraient présents en classe mais 


\section{eJRIEPS 32 avril 2014}

qu'ils ne participeraient pas à l'explication des consignes, à la distribution ou au ramassage des fiches documentaires et qu'ils n'auraient pas accès aux réponses des élèves. Après avoir récupéré le formulaire de consentement parental, nous avons présenté l'objectif de l'étude, expliqué comment remplir la fiche documentaire et souligné le caractère anonyme de l'exercice. Cette procédure a été uniforme pour toutes les classes rencontrées. Les élèves qui ne remplissaient pas la fiche documentaire étaient regroupés à l'écart et mis sous la responsabilité de l'enseignant pour ne pas qu'ils dérangent leurs camarades.

\subsection{Traitement et analyse des données}

Les données obtenues avec la première partie de la fiche documentaire ont été traitées de manière descriptive sous forme de fréquences absolues et relatives, de moyennes et d'écarts-types. Les données obtenues avec la deuxième partie de la fiche documentaire, celle concernant les incidents critiques rapportés par les élèves, ont d'abord été retranscrites sur support informatique avant de faire l'objet d'une analyse de contenu thématique selon une approche inspirée à la fois de Cresswell (1994) et de L'Écuyer (1990). Cette analyse a été réalisée en six étapes. La première a consisté en la lecture des récits racontés par les élèves. La deuxième a cherché à faire émerger, de manière inductive, de grandes catégories en regroupant les incidents critiques selon des thèmes compatibles. La troisième étape a consisté à relire les incidents critiques regroupés dans chaque catégorie pour formaliser les catégories et les sous-catégories. Une grille de codage contenant un titre, une définition et un code numérique pour chaque catégorie et chaque sous-catégorie a alors été élaborée. Dans une quatrième étape, nous avons vérifié la robustesse de la grille de codage en testant la fidélité intrajuge et interjuges de chaque catégorie à l'aide de la formule suivante tirée de Fortin (2010): \% accord = Nombre d'accords / Nombre total d'observations X 100. Nous avons pour cela sélectionné au hasard un échantillon de 50 incidents critiques (13,5\% du corpus) que nous avons placés dans les catégories et les sous-catégories déjà élaborées. Sept jours plus tard, tous $(100,0 \%)$ des incidents critiques ont été reclassés conformément à la classification initiale par un même codeur. Un autre chercheur, expert en analyse de contenu, a ensuite classé à son tour ces mêmes 50 incidents critiques à l'aide de la grille de codage. Quarante-quatre incidents critiques (88,0 \%) ont été regroupés de façon conforme à la classification établie par le premier codeur. L'accord interjuges a été jugé satisfaisant. Dans une cinquième étape, nous avons procédé à des ajustements des définitions des 


\section{eJRIEPS 32 avril 2014}

éléments de la grille. La sixième et dernière étape a consisté à reprendre de même qu'à finaliser le codage de l'ensemble du corpus recueilli.

\section{Résultats}

Cette étude avait pour objectif d'identifier et de hiérarchiser les inducteurs des émotions positives et négatives des élèves en EPS. Trois cent soixante-neuf incidents critiques ont été obtenus. De ce nombre, $13(3,5 \%)$ ont été rejetés parce qu'ils n'étaient pas assez exhaustifs ou ne répondaient pas à la consigne donnée aux élèves, empêchant ainsi leur classement. L'analyse inductive des 356 incidents critiques restant nous a permis d'élaborer une taxonomie composée de huit catégories d'éléments déclencheurs d'émotions positives et/ou négatives. En outre, nous avons identifié au sein de huit incidents critiques deux sources émotionnelles de même importance. Chacun de ces huit incidents critiques a donc été placé dans deux catégories distinctes, rehaussant à 364 le nombre d'incidents critiques répartis dans les catégories. Les huit catégories identifiées correspondent : (1) à la perception de l'enseignant; (2) aux réussites et échecs personnels consécutifs à la pratique des APSA; (3) à la perception du rapport aux pairs; (4) aux émotions et sensations liées à la pratique des APSA; (5) au désaccord avec les prescriptions d'évaluation nationales ; (6) à l'absence de participation à la pratique des APSA; (7) au contexte matériel de pratique des APSA et (8) à la participation alternative en cours d'EPS. Chacune de ces catégories se subdivise en deux sous-catégories : une rapportant des émotions positives et une rapportant des émotions négatives (Tableau 2).

Tableau II. Répartition des 364 incidents critiques obtenus des élèves dans les huit catégories d'inducteurs émotionnels

\begin{tabular}{|c|c|c|c|c|c|c|}
\hline & \multicolumn{2}{|c|}{$\begin{array}{l}\text { Nombre d'incidents } \\
\text { critiques relatant un } \\
\text { évènement positif }\end{array}$} & \multicolumn{2}{|c|}{$\begin{array}{l}\text { Nombre d'incidents } \\
\text { critiques relatant un } \\
\text { évènement négatif }\end{array}$} & \multicolumn{2}{|c|}{ TOTAL } \\
\hline & $f$ & $f \%$ & $f$ & $f \%$ & $f$ & $f \%$ \\
\hline Perception de l'enseignant & 91 & 25,0 & 55 & 15,1 & 146 & 40,1 \\
\hline $\begin{array}{l}\text { Réussites et échecs personnels } \\
\text { consécutifs à la pratique des APSA }\end{array}$ & 59 & 16,2 & 13 & 3,6 & 72 & 19,8 \\
\hline Perception du rapport aux pairs & 52 & 14,3 & 14 & 3,8 & 66 & 18,1 \\
\hline $\begin{array}{l}\text { Emotions et sensations liées à la } \\
\text { pratique des APSA }\end{array}$ & 19 & 5,2 & 45 & 12,4 & 64 & 17,6 \\
\hline $\begin{array}{l}\text { Désaccord avec les prescriptions } \\
\text { d'évaluation nationales }\end{array}$ & 0 & 0,0 & 9 & 2,5 & 9 & 2,5 \\
\hline $\begin{array}{l}\text { Absence de participation à la pratique } \\
\text { des APSA }\end{array}$ & 3 & 0,8 & 1 & 0,3 & 4 & 1,1 \\
\hline $\begin{array}{l}\text { Contexte matériel de pratique des } \\
\text { APSA }\end{array}$ & 0 & 0,0 & 2 & 0,5 & 2 & 0,5 \\
\hline $\begin{array}{l}\text { Participation alternative en cours } \\
\text { d'EPS }\end{array}$ & 1 & 0,3 & 0 & 0,0 & 1 & 0,3 \\
\hline TOTAL & 225 & 61,8 & 139 & 38,2 & 364 & 100 \\
\hline
\end{tabular}




\section{eJRIEPS 32 avril 2014}

Les incidents critiques contenus au sein de chaque sous-catégorie ont ensuite été finement classifiés jusqu'à un fin niveau de granulation. Au regard du nombre d'incidents critiques obtenus par catégorie, nous axerons les résultats et leur discussion sur les quatre premières catégories (Tableau 2), avec une focale sur la première, intitulée «Perception de l'enseignant», qui rassemble presque la moitié des incidents critiques obtenus.

3.1. Incidents critiques ayant trait à la perception de l'enseignant

L'analyse descriptive des incidents critiques réalisée à l'aide de quasi-statistiques (Maxwell, 2005) ayant trait à la perception de l'enseignant a établi que ce dernier constitue la source des émotions de 146 élèves, soit 40,10 \% du nombre total d'élèves participant à l'étude. Cette part ne varie guère selon la valence émotionnelle de l'évènement raconté par les élèves. La perception de l'enseignant d'EPS et de ses actions constitue donc très nettement le plus important inducteur des émotions des élèves interrogés en EPS.

La figure 1 montre que $60,4 \%$ des incidents critiques positifs font référence à des comportements interpersonnels de l'enseignant à l'égard des élèves. Par ailleurs, presque $30 \%(28,6 \%)$ des élèves évoquant l'enseignant le font sous l'angle des activités et des exercices qui leur sont proposés en EPS. Enfin, 10 élèves (11\%) ont déclaré avoir ressenti des émotions positives consécutivement au mode de groupement qui leur a un jour été proposé par leur enseignant. 


\section{eJRIEPS 32 avril 2014}

Figure 1. Schéma organisateur des incidents critiques positifs classés dans la catégorie "Perception de l'enseignant » et dans les sous-catégories associées.

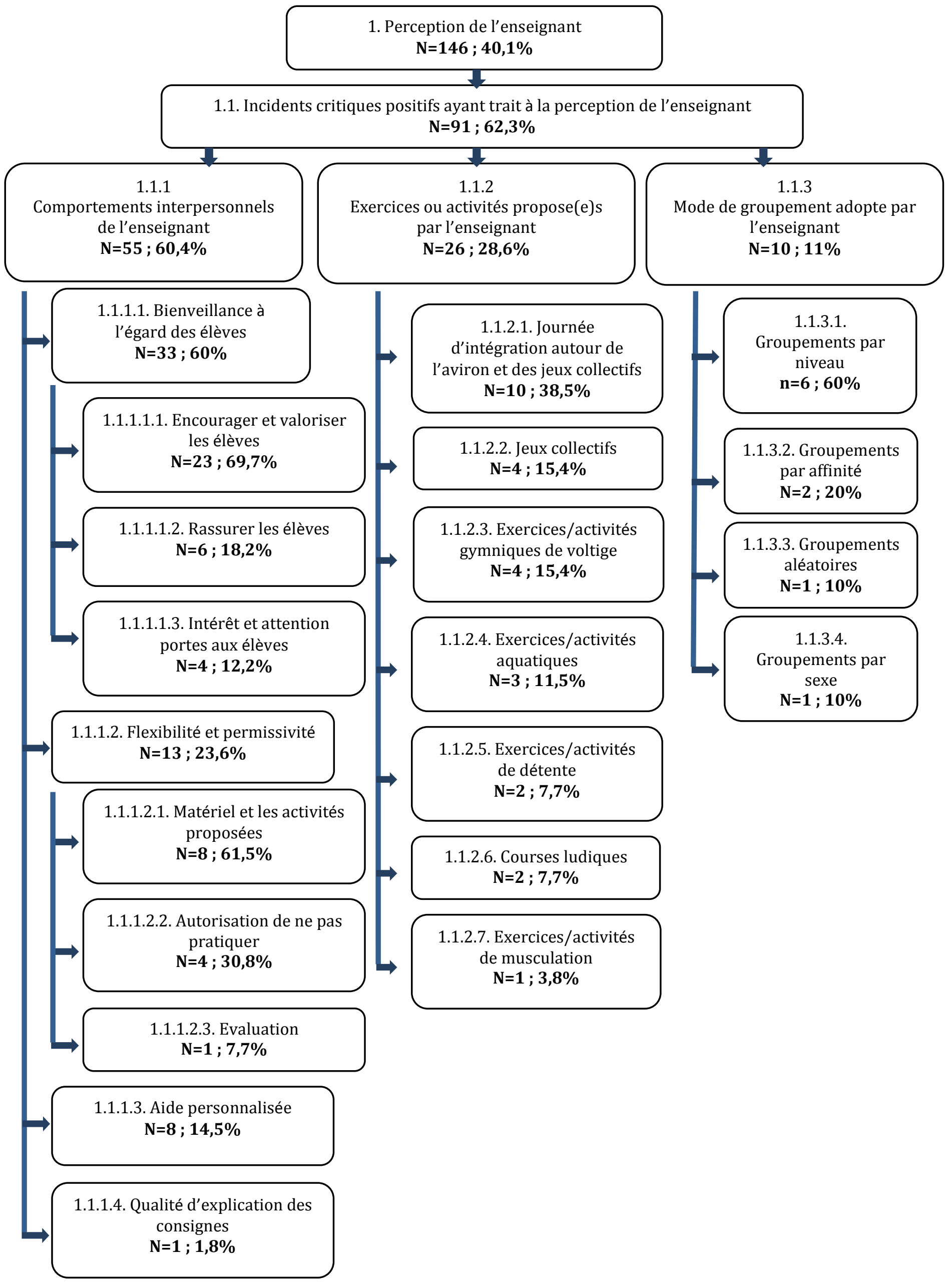




\section{eJRIEPS 32 avril 2014}

La figure 2 révèle que près de la moitié des élèves $(49,1 \%)$ déclarant avoir ressenti des émotions négatives en EPS du fait de l'enseignant évoquent les comportements interpersonnels de ce dernier. En outre, 15 élèves $(28,6 \%)$ ont parlé des émotions négatives qu'ils ont un jour ressenties en EPS en raison de leur désaccord avec l'évaluation de l'enseignant. Pour finir, un peu plus de $20 \%$ (21,8\%) des élèves évoquant les émotions négatives qui ont un jour été générées par leur enseignant situent l'inducteur de leurs émotions au plan des activités et exercices proposés par l'enseignant ou du mode de groupement choisi par ce dernier. 


\section{eJRIEPS 32 avril 2014}

Figure 2. Schéma organisateur des incidents critiques négatifs classés dans la catégorie «Perception de l'enseignant » et dans les sous-catégories associées.

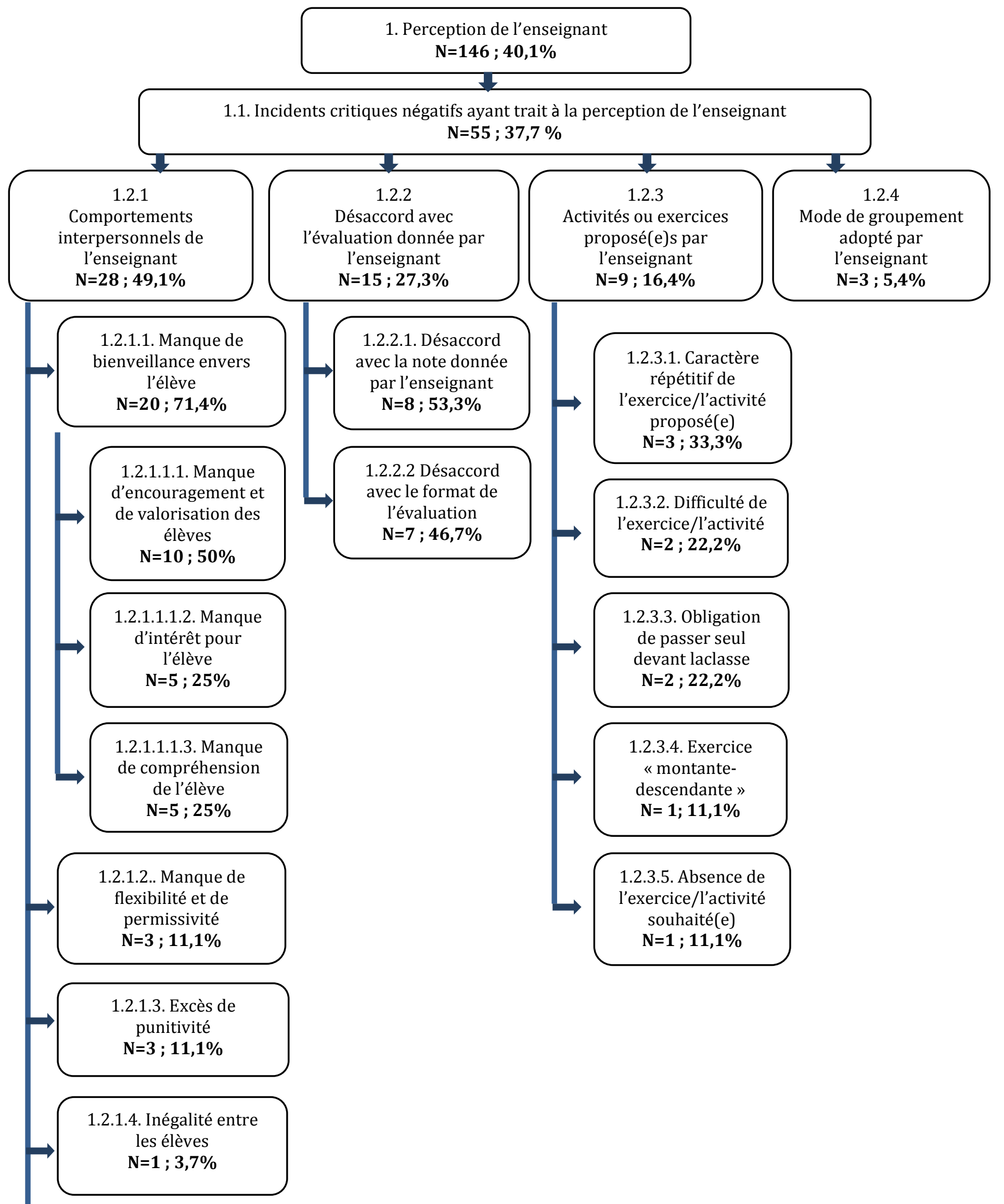




\section{eJRIEPS 32 avril 2014}

Ce sont les comportements interpersonnels de l'enseignant qui génèrent le plus souvent les émotions positives ou négatives dont parlent les élèves. Les élèves qui évoquent les comportements interpersonnels de l'enseignant sont particulièrement marqués par la bienveillance de celui-ci à leur égard, des encouragements et de l'intérêt qu'il leur porte. Une élève déclare par exemple :

Etant asthmatique et donc peu endurante, très peu de professeurs m'encourageaient. Ils n'étaient pas satisfaits. En revanche, cette année pour la première fois ma prof. m'a encouragée, elle est positive, nous incite à donner le meilleur de soi-même, j'ai retrouvé ma confiance en moi grâce à elle (élève 9, C1 Justine).

Par ailleurs, l'absence d'encouragements ou des propos perçus comme dévalorisants peuvent générer des émotions très négatives chez les élèves et ultimement porter atteinte à leur engagement en EPS :

C'était quand on faisait de l'athlétisme, il fallait courir au moins 10 minutes. Moi ça faisait longtemps que je n'avais pas couru et d'autres personnes aussi du coup on n'a pas réussi. Notre prof. était pas content et il nous a dit en gros qu'on finirait notre vie en fauteuil roulant ou qu'on aurait des problèmes vers 50 ans. Selon moi, ça n'a rien à voir, ce n'est pas parce qu'on réussit pas quelque chose qu'on a raté notre vie. Je trouvais ça pas super encourageant, parce qu'on nous prend un peu pour des moins que rien (élève 21, C1, Stéphane).

Si les élèves n'ont pas été nombreux à commenter la capacité de leur enseignant à expliquer les consignes, un élève raconte toutefois :

On était à la piscine et le professeur nous expliquait les consignes. Je trouvais qu'il nous expliquait pas très bien et c'était pas cool. II ne prenait même pas quelqu'un pour montrer l'exemple, je trouvais ça pas bien. C'est dur d'être tout le temps content quand on nous fait faire des choses difficiles (élève 22, C1, Stéphane).

Les incidents critiques positifs et négatifs se référant à la perception de l'enseignant ont également souvent trait à la manière dont l'enseignant didactise les APSA. Les incidents critiques regroupés dans cette catégorie se réfèrent donc non pas à la nature de l'APSA pratiquée mais bien à des tâches particulières, proposées à l'initiative de l'enseignant. Leur contenu suggère une volonté de la part des élèves d'être confrontés à des activités originales et ludiques. Trois élèves ont dénoncé le caractère répétitif de certains exercices 


\section{eJRIEPS 32 avril 2014}

proposés par leur enseignant, d'autres ont raconté avoir apprécié pratiquer des activités de détente telles que la sophrologie ou la relaxation, relativement peu proposées en EPS, ainsi que des jeux collectifs et des courses ludiques. Plusieurs ont évoqué des exercices aquatiques tels qu'aller chercher des mannequins dans l'eau ou encore des exercices de voltige consistant par exemple à réaliser des figures en trampoline.

Plusieurs élèves participant à l'étude ont quant à eux raconté avoir ressenti des émotions très positives à l'occasion d'une journée d'intégration organisée par leur enseignant au début de l'année scolaire et consistant à passer la journée à pratiquer l'aviron et à faire des jeux collectifs.

Au début de l'année, notre professeure d'EPS a organisé une «journée d'intégration " durant laquelle nous avons pratiqué l'aviron avec la classe. J'ai donc pagayé conjointement avec mes camarades de classe. J'étais heureuse à la fois de pratiquer ce sport mais aussi de le pratiquer avec des amis, je me suis beaucoup amusée tout au long de la journée (élève 28, C2, Justine).

Outre le nombre important d'occurrences recueillies dans cette catégorie, certains élèves attribuent même à leur enseignant un réel pouvoir de motivation pour la pratique des APSA, suggérant l'influence importante qu'il peut exercer sur leurs apprentissages : « Je déteste aller à la piscine, mais depuis que j'ai cette professeure, je prends plaisir à y aller. C'est quelqu'un d'adorable qui donne envie de travailler» (élève 25, C1, Justine). De manière indéniable, l'expérience de l'enseignant peut constituer une variable majeure influençant le choix de l'évènement raconté par les élèves. En effet, les élèves participant à cette étude sont placés sous la responsabilité de huit enseignants ayant une expérience de un à trente ans dans l'enseignement de l'EPS. Cet écart peut induire des variations dans la perception qu'ont les élèves de leur enseignant et des émotions qu'il induit chez eux.

3.2. Incidents critiques ayant trait aux réussites et aux échecs personnels consécutifs

à la pratique des APSA

L'analyse descriptive des incidents critiques ayant trait aux réussites et aux échecs personnels consécutifs à la pratique des APSA révèle que les élèves ont été presque $20 \%$ $(19,8 \%)$ à avoir été marqués positivement ou négativement par une réussite ou un échec vécu en EPS depuis le début de l'année scolaire. Plus précisément, les élèves sont 59 $(16,2 \%)$ à évoquer une réussite marquante en EPS et $13(3,6 \%)$ à raconter un échec vécu en cours. 


\section{eJRIEPS 32 avril 2014}

Par ailleurs, les réussites racontées par les élèves ont eu lieu presque quatre fois plus souvent lors de la pratique d'une APSA individuelle. Par exemple, l'un d'eux nous confie :

En EPS lorsque j'ai gagné la course d'athlétisme au stade j'ai ressenti des émotions positives. La course était serrée jusqu'au bout mais j'ai tout de même gagné grâce aux sports que je fais hors des cours. J'étais fier de moi et encore plus motivé à améliorer ma vitesse de course (élève 6, C1, Stéphane).

Treize élèves ont tout de même évoqué une réussite personnelle vécue dans le cadre d'une APSA collective, notamment en volley-ball.

Si nous nous penchons à présent sur les échecs vécus par les élèves en cours d'EPS, nous observons que plus de $90 \%(92,3 \%)$ de ces échecs sont survenus lors de la pratique d'une APSA individuelle. La moitié d'entre eux ont eu lieu en natation ainsi qu'en athlétisme. Par exemple, un élève rapporte l'évènement suivant :

C'était pendant le cours de piscine on devait faire des groupes de deux et je me suis retrouvée avec une fille qui était beaucoup plus forte que moi et comme j'étais aussi très fatiguée et que je n'y arrivais pas j'ai eu l'impression d'être nulle. J'étais très fatiguée, donc je n'y arrivais pas, j'ai eu l'impression d'être nulle et ça m'a rendue triste (élève 6, C1, Alisée).

3.3. Incidents critiques ayant trait à la perception du rapport aux pairs L'analyse descriptive des incidents critiques traitant de la perception du rapport aux pairs a établi que presque $20 \%(18,1 \%)$ des élèves ont déclarés avoir été marqués par un évènement mettant en cause leurs camarades en EPS. Une très large part de ces élèves $(78,8 \%)$ a évoqué les émotions positives générées par cette relation aux pairs. Plus précisément, 88,5 \% des élèves ayant été positivement marqués par leur relation aux pairs ont relaté un évènement survenu dans le cadre de la pratique des APSA, qu'il s'agisse de moments conviviaux ou d'épisodes en lien avec le travail scolaire. Vingt élèves sur les 46 évoquant un moment collectif vécu dans le cadre de la pratique des APSA déclarent avoir ressenti des émotions positives suite à une réussite collective ou après avoir constaté la solidarité avec leurs camarades. Par exemple, un élève raconte :

Durant l'athlétisme il fallait courir en un temps donné une certaine distance. Durant cet exercice j'ai énormément apprécié la solidarité qui s'est dégagée car on s'encourageait lorsque l'un d'entre nous craquait, c'était plutôt agréable et il y avait donc une bonne entente et de la solidarité. J'ai trouvé ça super sympa. Cela nous encourageait. Ca a créé une bonne ambiance. C'était très motivant (élève 14, C1, Jocelyne). 


\section{eJRIEPS 32 avril 2014}

Toutefois, l'essentiel des incidents critiques évoquant le rapport aux pairs rapporte un moment de convivialité vécu en groupe, dans la pratique des APSA mais sans avoir pour objet le travail scolaire ou l'apprentissage. La description quantitative révèle en outre que ces moments de convivialité sont majoritairement vécus en course d'orientation, souvent pratiquée collectivement. Par exemple, une élève nous dit :

C'était au début de l'année, en septembre, nous étions en course d'orientation, nous avions fait plusieurs groupes de 2 et j'étais en binôme avec une très bonne amie. II faisait chaud, nous étions aux Gayeulles et nous courrions à travers les allées en chantant, c'était très amusant. C'était vraiment amusant nous étions en "communion» l'une avec l'autre, c'était un très bon moment (élève 18, C1, Jean).

Les sports collectifs sont également le théâtre de moments conviviaux entre élèves, puisque 12 élèves $(46,1 \%)$ racontent avoir vécu de tels moments en basket ou en volley. Ces épisodes racontés par les élèves n'ont pas directement pour fin le travail et la progression au plan scolaire.

Par ailleurs, 21,2\% des élèves ayant évoqué un incident critique en lien avec les pairs ont affirmé avoir ressenti des émotions négatives. Nous constatons que la plupart de ces élèves $(78,6 \%)$ situent cet évènement dans le cadre de la pratique des APSA. Par ailleurs, trois élèves $(21,4 \%)$ déclarent que leurs émotions négatives sont nées du comportement de leurs pairs dans les vestiaires. Les résultats révèlent qu'au sein des 78,6 \% d'élèves rapportant un évènement vécu dans la pratique des APSA, 21,2 \% ont évoqué les désagréments qu'ils ont causés à un de leur camarade, ou plus souvent, qu'un ou plusieurs camarades leur ont causés. Une élève déclare par exemple :

Une fois pendant une séance de volley j'avais des difficultés à rattraper les balles et des élèves de mon équipe ont décidé de m'envoyer hors du terrain et de se répartir les places comme si je ne faisais pas partie de l'équipe parce que je n'étais pas assez forte. J'étais furieuse d'être exclue à cause de mon niveau (élève 2, C1, Léa).

Plus encore, trois élèves $(21,4 \%)$ ont fait état de violences physiques dont eux ou des camarades auraient été l'objet dans les vestiaires. Parce qu'ils constituent un lieu où peut naitre une certaine impunité des élèves, l'enseignant n'y pénétrant pas, les vestiaires semblent propices aux agressions. Un élève confie par exemple :

Dans les vestiaires en EPS en début d'année je me faisais traiter de tous les noms du coup je me faisais frapper cela me rendait triste et même en dehors du 


\section{eJRIEPS 32 avril 2014}

cours d'EPS ça m'arrivait mais la prof était là pour m'aider dans mes problèmes et là ça s'est amélioré » (élève 20, C2, Alisée).

3.4. Incidents critiques ayant trait aux émotions et aux sensations liées à la pratique des APSA

L'analyse des 64 incidents critiques $(17,6 \%)$ traitant des émotions et des sensations vécues dans la pratique des APSA suggère que les élèves ont été plus nombreux à se dire négativement marqués par la pratique des APSA, puisque 70,3\% des incidents critiques regroupés dans cette catégorie relatent des émotions négatives. Parmi eux, $71,1 \%$ des élèves ont témoigné d'un malaise consécutif à la pratique. Douze élèves $(26,7 \%)$ évoquent quant à eux les émotions négatives en lien avec la programmation des APSA qui leur a été proposée durant l'année. Enfin, un incident critique (2,2\%) rapporte des émotions négatives que la pratique des APSA a créées par le rappel d'un évènement déplaisant vécu antérieurement par l'élève.

Malgré tout, deux élèves (10,5\%) ont déclaré avoir ressenti des émotions positives parce que le fait de pratiquer une APSA leur a rappelé des souvenirs plaisants vécus antérieurement. Aussi, 19 élèves $(29,7 \%)$ ont déclaré avoir ressenti des émotions positives en lien avec la pratique des APSA. Parmi ceux-ci, 17 incidents critiques (89,5\%) font référence aux émotions positives générées par la pratique de l'APSA. Une élève déclare par exemple :

C'était à la piscine. Je nageais tranquillement et d'habitude, je stresse quand je suis à la piscine, je me suis détendue et soudain, j'ai ressenti une sensation de plénitude comme jamais je n'avais ressenti auparavant. C'est difficile à expliquer mais c'était vraiment agréable car j'étais vraiment détendue (élève 16, C1, Justine).

II semble que d'une manière générale, les activités plus atypiques, telles que le kayak ou la course d'orientation, soient plus facilement acceptées par les élèves. Aucun d'entre eux n'a mentionné une insatisfaction de voir ces activités inscrites au programme de l'année. Seulement, c'est notamment dans la pratique de ces activités de pleine nature que les élèves dénoncent les désagréments. Par exemple, un élève nous raconte :

Au début de l'année on a fait course d'orientation, et on était par deux. J'étais avec une copine et on est tombé dans de la vase près de l'étang ! On était super mal et on en avait partout! J'étais plutôt en colère. Je trouve ça dangereux de nous laisser comme ça dans des endroits où ça aurait pu tourner mal. J'étais vraiment en colère, déçu, triste et fatigué ! J'en avais 


\section{eJRIEPS 32 avril 2014}

assez ! Faire tout ça pour du sport! C’est vraiment pas super! (élève 1, C1, Jean)

Un autre élève évoque quant à lui la pratique du kayak lors de conditions météorologiques difficiles :

Lors des cours de kayak, quand il faisait beau au début de l'année, tout se passait bien. Le cours était original, mais lors de la fin du cycle, le temps s'était vraiment dégradé et on a dû faire le contrôle en short-combi, plein d'eau alors qu'il pleuvait et faisait froid. Je suis même tombé dans l'eau. C'était un cours horrible (élève 8, C2, Stéphane).

Nous comprenons donc que la programmation des APSA proposées aux élèves au cours de l'année est susceptible de fortement influencer les inducteurs émotionnels évoqués par les élèves, qui ont été amenés à raconter un évènement marquant depuis le début de l'année scolaire.

En outre, lorsqu'ils évoquent les désagréments générés par la pratique des APSA, les élèves évoquent fréquemment les conséquences d'un effort important dont ils ont fait preuve durant cette pratique.

Outre les quatre catégories exposées et discutées précédemment, l'analyse descriptive des incidents critiques recueillis a révélé que neuf élèves expriment leur désaccord avec les prescriptions d'évaluation nationales, quatre font référence à une absence de participation au cours d'EPS, deux déplorent les conditions matérielles de pratique des APSA, et un évoque les émotions positives ressenties lors d'une participation alternative au cours d'EPS.

\section{Discussion}

Les perceptions d'élèves recueillies au cours de cette étude peuvent être discutées au regard des résultats déjà exposés dans la littérature. Si l'absence d'un recueil des perceptions de l'enseignant ne nous permet pas d'émettre de préconisations à destination de l'activité d'enseignement, la confrontation des résultats obtenus au cours de cette étude avec les recommandations déjà formulées dans la littérature nous autorise toutefois à rediscuter de la prise en compte de l'activité émotionnelle des élèves en classe.

4.1. L'influence majeure de l'enseignant sur les émotions exprimées par les élèves II apparait au regard de ces résultats que le travail de l'enseignant n'est pas seulement de nature intellectuelle mais aussi émotionnelle, car il faut mobiliser, outre les savoirs académiques, des connaissances, des savoir-faire et des savoir-être divers pour assurer 


\section{eJRIEPS 32 avril 2014}

des interactions qui rendent possibles les apprentissages (Rayou \&Van Zanten, 2004). Cette influence particulièrement importante des comportements interpersonnels sur les émotions exprimées par les élèves recoupe les résultats obtenus par Wubbels et Levy (1993). Les auteurs établissent que la perception des comportements interpersonnels de l'enseignant par les élèves est très importante, dans le sens des propos de Gordon (1979) qui affirme qu'un intervenant, quel que soit ce qu'il veut enseigner, doit avant tout tenir compte de ses relations personnelles avec les élèves. II est aussi bien documenté que l'enthousiasme de l'enseignant joue un rôle crucial vis-à-vis des attitudes des élèves, de leur motivation à l'école et de leur accomplissement (Brophy \& Good, 1974 ; Siedentop, 1994). Récemment, partant de travaux menés au Québec auprès de 90 enseignants d'EPS et de 2000 élèves du secondaire, Dumas (2009) et Dumas, Desbiens, Spallanzani et Colin (2012) ont mis en évidence que plus les élèves perçoivent leur enseignant comme étant enthousiaste, plus il est vu comme soutenant, capable d'exercer du leadership, de faire preuve de bienveillance et de permissivité. À l'opposé, les élèves qui perçoivent leur enseignant comme « Jamais » ou «Parfois » enthousiaste tendent aussi à le considérer peu soutenant, très dominant et très exigent. Néanmoins, plusieurs recherches centrées sur le climat de classe font état d'un climat émotionnel plutôt neutre en classe, l'enseignant ne se montrant pas vraiment enthousiaste à l'égard des élèves (Brunelle, Spallanzani, Lord \& Petiot, 1983 ; Ward, 1982 ; Siedentop, 1994). Finalement, plus les enseignants sont jugés coopératifs, plus les attitudes des élèves sont favorables à l'apprentissage. Aussi, plus l'enseignant est dominant, plus les élèves réussissent (Lapointe \& Legault, 1999). Au travers de notre étude, la notion de contrôle transparait dans le leadership perçu chez l'enseignant (Dumas, Desbiens, Spallanzani \& Colin, 2012), plus particulièrement dans l'incident critique évoquant l'efficacité de l'enseignant à expliquer les consignes. L'étude de Jeantheau et Murat (1998) a pourtant révélé qu'aux yeux des élèves, le professeur « idéal » était avant tout un enseignant qui savait expliquer pour $90 \%$ des répondants. II semble que les élèves participant à notre étude n'aient pas été suffisamment marqués par de tels comportements pour choisir de nous les rapporter.

Nous avons également pu constater précédemment que sur neuf élèves évoquant positivement le mode de groupement proposé par l'enseignant, six rapportent un épisode durant lequel ce dernier s'est appuyé sur le niveau des élèves pour former les groupes. L'incident critique précédent suggère toute la nécessité de penser la formation des groupes de travail de manière à contrôler la genèse d'émotions négatives chez les élèves. Comme l'expliquent Pekrun et Stephens (2012), le contexte de l'apprentissage induit une 


\section{eJRIEPS 32 avril 2014}

multitude d'émotions sociales qui peuvent même se superposer aux émotions de réussite (achievement).

Les résultats obtenus au cours de cette étude nous invitent également à discuter l'importance de la manière dont l'enseignant didactise les APSA pour générer le plaisir des élèves dans la pratique. Pekrun et Stepens (2012) insistent sur le fait que les émotions associées aux activités (Topic emotions) peuvent fortement influencer l'engagement en affectant l'intérêt et la motivation pour une activité donnée. De son côté, Parlebas (2005) dit qu'il n'est pas vain de « reludiser » les APSA, c'est à dire de favoriser l'apparition d'un plaisir spontané et d'une motivation joyeuse, sans nécessairement de finalité utilitaire. Malgré tout, les fondements culturels de chaque APSA ne doivent pas être ignorés pour favoriser l'accès des élèves à une culture raisonnée, critique et réfléchie des pratiques sociales de référence et des valeurs qu'elles véhiculent (Programmes EPS lycées, 2010). Ce constat nous incite à encourager la conduite de recherches touchant aux situations d'apprentissage pouvant allier la progression des élèves et leur plaisir d'agir. En EPS, un certain nombre de praticiens et de chercheurs se sont attachés à produire de tels travaux, à l'image de Leveau, Louis et Sève (1999), qui ont démontré l'intérêt des « matchs à thème » pour permettre aux élèves d'intégrer des acquisitions techniques et tactiques dans un contexte suscitant un engagement affectif fort lié à la confrontation à l'adversaire. Plus récemment et d'un point de vue professionnel, Monnet (2009) a développé le « Cartaping », un jeu basé sur l'utilisation de cartes « pouvoir » et destiné à rendre attrayante et efficace la pratique du tennis de table.

Plus généralement, lorsque l'on sait que la journée d'intégration n'a été proposée qu'à deux classes sur les seize participants à l'étude et que dix élèves ont choisi de la raconter, nous comprenons que ce type d'évènement possède un poids tout particulier dans les émotions ressenties par les élèves. De surcroit, si réellement les perceptions d'un individu tendent à rester stables au cours du temps (Dweck, 1999), ce type d'évènement peut contribuer à cristalliser des relations positives avec l'enseignant et une cohésion entre les camarades dès la rentrée, contribuant ainsi à débuter l'année sur de bonnes bases.

4.2. L'influence des réussites et des échecs vécus en EPS sur les émotions exprimées par les élèves

Cette catégorie correspond à ce que Pekrun et Stepens (2012) appellent les émotions associées aux résultats (outcome emotions). Elles incluent les émotions prospectives et rétrospectives associées à des épisodes de succès et d'échecs. Dans cette étude, il n'est question que du second type d'émotion. S'il n'est pas pour autant possible de conclure 


\section{eJRIEPS 32 avril 2014}

que, d'une manière générale, le cours d'EPS est davantage source de réussites que d'échecs, les incidents critiques regroupés dans cette catégorie ont davantage décrit une réussite qu'un échec. Une explication pourrait consister à dire que les enseignants ont peut-être eu le souci, notamment par le discours et l'établissement de relations empreintes de compréhension et de soutien, d'atténuer l'impact émotionnel négatif des échecs et de renforcer la fierté et le plaisir liés à la réussite. Toutefois, il se peut également que les élèves, consciemment ou non, se soient montrés réticents à rapporter une anecdote ayant concouru à diminuer leur estime de soi. En effet, d'après la théorie de « mise en valeur du soi 》 (self-enhancement theory), les individus seraient enclins à avoir un jugement positif d'eux-mêmes (Jussim, Yen \& Aiello, 1995), expliquant qu'ils réagissent davantage et soient plus marqués par des moments vécus de manière positive pour leur image. Ils peuvent ainsi tout aussi bien avoir évacué de leur esprit les échecs qu'ils ont vécus en EPS.

Par ailleurs, les réussites racontées par les élèves ont eu lieu presque quatre fois plus souvent lors de la pratique d'une APSA individuelle. Nous faisons l'hypothèse que pratiquer les APSA en groupe tend à masquer les réussites ou les échecs personnels. Une réussite ou un échec personnel(le) en sport collectif peut être expliqué(e) par un fonctionnement réussi ou non du groupe, inhibant alors le mérite ou le tort de l'élève personnellement impliqué. En outre, il se peut que le faible nombre de réussites marquantes racontées par les élèves s'explique par la diminution de leurs efforts fournis en situation groupale. Ce phénomène de paresse sociale (Harkins, Latané \& Williams, 1980) survient notamment quand l'individu a l'impression que sa prestation n'est pas reconnue, notamment par l'enseignant, et qu'il diminue ses efforts fournis (Harkins, 1987). La possibilité qu'il vive des réussites personnelles marquantes est alors moindre. Dans cette optique, il est recommandé de différencier les performances individuelles des performances collectives, que ce soit dans la notation ou dans les tâches attribuées aux élèves. II s'agit de créer une dynamique de groupe par la fixation d'un but commun mais aussi une interdépendance entre les membres et des rôles dévolus à chacun (Carron, Colman, Wheeler \& Stevens, 2002). Si l'élève se sent davantage impliqué dans la réussite du groupe, il consentira en outre plus facilement à fournir autant d'efforts que s'il avait eu à réaliser individuellement la tâche.

Concernant les échecs exprimés par les élèves, nous avons pu observer la fréquence de ces échecs dans des APSA individuelles. Ce résultat nous incite à envisager l'importance d'une responsabilisation de l'élève pour qu'il continue de fournir les efforts nécessaires à 


\section{eJRIEPS 32 avril 2014}

sa progression. Ce n'est en effet pas l'évènement en lui-même qui détermine le comportement futur de l'individu mais les explications qu'il en donne (Weiner, 1985). Plus encore, le caractère résolument social des émotions vécues dans le contexte de la classe (Visioli, Petiot \& Ria, soumis) suggère le poids de la présence des pairs et de l'enseignant lors des échecs vécus par les élèves. Comme l'ont noté Newton, Duda, \& Yin (2000), la comparaison sociale, les récompenses, les punitions, mais aussi la place accordée à l'effort par l'intervenant constituent les éléments distincts du climat motivationnel. Ces éléments sont influencés par l'enseignant, ses interprétations et la manière dont les élèves les perçoivent (Watson \& Clocksin, 2013).

4.3. L'influence du rapport aux pairs sur les émotions exprimées par les élèves L'essentiel des incidents critiques évoquant le rapport aux pairs rapporte un moment de convivialité vécu en groupe, dans la pratique des APSA mais sans avoir pour objet le travail scolaire ou l'apprentissage. Si l'approche adoptée au cours de cette recherche se distingue grandement des méthodologies employées par les chercheurs en psychanalyse, nous sommes d'avis que ce résultat peut être mis en lien avec l'idée selon laquelle, durant l'adolescence, l'environnement devient une source majeure de stimulations et génère une infinité d'émotions difficiles à prévoir et à appréhender, projetant les élèves au cœur d'un «bain émotionnel » (Braconnier, 1998). D'un point de vue social, chacun entreprend à cette période un véritable dialogue émotionnel avec autrui (Therme, 1995). Les camarades de classe ainsi que l'enseignant s'imposent nécessairement comme des acteurs privilégiés qui entourent l'élève, influent sur ses émotions et sur son implication en cours. Comme l'ont montré empiriquement Gagnaire et Lavie (2005), les élèves cherchent à vivre en EPS des situations générant un plaisir gratuit, d'amusement avec les camarades, qui entre en décalage avec le plaisir cultivé par l'institution scolaire.

Ces épisodes racontés par les élèves n'ont pas directement pour fin le travail et la progression au plan scolaire. Ils peuvent néanmoins s'avérer pertinents pour maintenir un climat d'apprentissage favorable en EPS, de sorte qu'il puisse être intéressant de les instrumentaliser en proposant des séquences de travail collectives régulières. Ces séquences de travail pourraient être incluses à l'échauffement dans le cas des APSA individuelles sans empêcher les élèves de vivre individuellement leur pratique de manière à ce que leur engagement ne soit pas tributaire de celui des autres (Carron et al., 1982). Pour finir, si les désagréments liés aux blessures ne peuvent être totalement écartés d'une discipline qui possède comme objet le corps et l'activité physique, d'autres désagréments, de l'ordre du conflit, doivent attirer notre attention. Nos résultats rappellent en effet 


\section{eJRIEPS 32 avril 2014}

l'importance de sensibiliser les élèves à des valeurs fondamentales comme le respect et la tolérance, mais aussi de ne pas négliger l'importance de la vie sociale en classe. L'utilisation de sociogrammes (Moreno, 1943), qui mettent en évidence les leaders, les sous-groupes ainsi que les attraits ou les hostilités, peut par exemple permettre aux enseignants de saisir la dynamique de groupe instaurée dans les classes et d'identifier d'éventuels élèves isolés et d'orienter la formation des groupes en EPS.

\subsection{L'influence de l'APSA enseignée sur les émotions exprimées par les élèves}

Les émotions positives exprimées par certains élèves consécutivement à la pratique des APSA s'apparentent à un état de Flow (Csikszentmihalyi, 1975), qui survient lorsque l'individu est tendu vers le fonctionnement optimal du corps et de l'esprit, et que la tâche est accomplie pour elle-même. Lorsque nous connaissons les bienfaits du plaisir pour l'engagement durable dans la pratique des APSA (Perrin, 1993), nous saisissons toute l'importance de cultiver cet état de Flow en EPS. Le défi pour l'intervenant consiste à proposer des exercices qui se situent dans la zone de difficulté optimale de l'élève, aussi appelée la zone de délicieuse incertitude (Brunelle et al., 1988), qui cause la dissonance chez l'apprenant et l'incite à s'engager dans l'exécution de la tâche, pour relever le défi. Si la tâche est trop difficile, elle suscitera l'angoisse chez l'élève, alors qu'elle génèrera plutôt de l'ennui si ce dernier n'éprouve aucune difficulté à la réaliser (Brunelle et al., 1988).

II semble au regard de nos résultats que d'une manière générale, les activités plus atypiques, telles que le kayak ou la course d'orientation, soient plus facilement acceptées par les élèves. Malgré tout, c'est également dans ces activités que se produisent la majorité des désagréments exprimés pas les élèves. Ainsi, s’il faut bâtir une didactique du risque et de l'émotion (Therme, 1995), il convient surtout de tenir compte du caractère ambivalent de l'expérience du risque qui peut autant être générateur d'émotions positives que négatives s'il est trop présent (Delignières, 1993). Si l'insatisfaction ne constitue pas forcément une émotion négative (Gagnaire \& Lavie, 2005), tout l'enjeu consiste à trouver un compromis pour faire naitre une certaine dissonance chez les élèves sans nuire à leur engagement dans la pratique. En escalade et d'un point de vue professionnel, Moreau (2006) propose une progressivité dans la manière de confronter les élèves au risque. II préconise d'expérimenter d'abord la modalité de grimpe horizontale, pour ensuite aller vers différentes hauteurs de piton. II s'agirait aussi, en escalade, d'amener les élèves à réaliser des chutes encadrées ou encore, en kayak, à leur proposer de se retourner dans l'eau. 


\section{eJRIEPS 32 avril 2014}

En outre, lorsqu'ils évoquent les désagréments générés par la pratique des APSA, les élèves évoquent fréquemment les conséquences d'un effort important dont ils ont fait preuve durant cette pratique. Tant dans le discours qu'il donne aux élèves que dans les situations qu'il propose, l'enseignant peut favoriser chez les élèves ce plaisir dans l'effort, manipulant ainsi avec réussite la dialectique satisfaction/insatisfaction. Les conclusions de Ubaldi et Philippon (2003) au plan pédagogique vont dans le sens d'un plaisir qui soit à l'origine d'une adaptation de l'élève et donc, de raisons d'apprendre. Selon eux, il s'agit de faire passer l'élève d'un plaisir dans sa logique conative, plaisir de s'éprouver ou de se perdre, à un plaisir dépassé, une joie de progresser, d'apprendre et de se transformer. C'est en favorisant ce type de plaisir en EPS que l'enseignant pourra d'ailleurs lier les mobiles des élèves, dirigés davantage vers l'amusement entre amis (Gagnaire \& Lavie, 2005) et ceux de l'institution scolaire et des législateurs, plus tournés vers un plaisir comme moyen d'atteindre l'apprentissage. Les fiches ressources pour les lycées (2011) suggèrent d'ailleurs qu'en natation de vitesse, les élèves de niveau 2 doivent être amenés à ressentir du plaisir à agir tout au long de la succession d'efforts.

Pekrun et Stephens (2012) ont réalisé une taxonomie tridimensionnelle des émotions liées à la réussite scolaire, affirmant que certaines émotions positives comme le plaisir vont de pair avec une activation favorable à l'engagement et à la réussite scolaire. Les auteurs montrent aussi que des émotions négatives comme la colère peuvent avoir pour origine la même activation, confirmant la complexité à appréhender la question des émotions et ses liens avec d'autres phénomènes comme la réussite. Si les résultats présentés ici sont centrés sur les émotions ressenties et n'ont pas vocation à extrapoler de quelconques liens avec la réussite en EPS, reste que tenter d'appréhender les émotions des élèves peut nous aider à comprendre comment limiter ces « ruses du quotidien » inventées par les élèves pour échapper au cours d'EPS (Liotard, 1997). La littérature en sciences de l'éducation montre d'ailleurs que pour les enseignants, créer un bon climat par l'établissement de relations de qualité constitue non seulement un but (Canisius-Kamansi, Lessard, Riopel, Blais, Larose, Wright \& Bourque, 2008), mais aussi un enjeu de premier plan. En effet, le rapport affectif évoluant dans le temps (Kennedy \& Kennedy, 2004), il constitue un levier permettant de soutenir l'engagement des élèves dans les tâches et, ultimement, la réussite du processus d'enseignement-apprentissage (Shernoff, 2012). 


\section{eJRIEPS 32 avril 2014}

\section{Conclusion}

Cette étude avait, partant de la perspective d'élèves du secondaire, pour premier objectif d'identifier les inducteurs des émotions positives et négatives ressenties en EPS. Elle visait ensuite à rendre compte de l'organisation hiérarchique de ces inducteurs établie sur la base de leur fréquence d'apparition dans les propos des élèves. La technique des incidents critiques utilisée pour collecter les données a permis d'explorer les émotions ressenties par 364 élèves en lien avec leur cours d'EPS de même qu'à montrer de manière inductive que des évènements ponctuels et d'apparence ordinaire peuvent influer sensiblement sur les émotions ressenties en cours d'EPS. Compte tenu du caractère exploratoire de cette recherche, cette technique s'est avérée très intéressante en raison de son caractère économique et du contrôle qu'elle permettait au participant d'exercer en choisissant de développer spontanément un incident positif ou négatif perçu comme significatif.

L'analyse de contenu conduite de manière systématique a mené à l'identification de huit inducteurs émotionnels dont quatre principaux. Le premier, nettement plus important puisqu'il compte 40,1 \% des énoncés touche la perception qu'ont les élèves de leur enseignant. Les trois autres concernent dans l'ordre les réussites et les échecs consécutifs à la pratique des APSA, la perception du rapport au pairs et les émotions et sensations liées à la pratique des APSA. Ces trois dernières dimensions ont une taille comparable puisqu'elles regroupent respectivement $19,8 \%, 18,1 \%$ et $17,6 \%$ des énoncés codés. Fait intéressant, pour les trois premières dimensions, les élèves ont choisi spontanément de décrire davantage de situations ayant provoqué des émotions positives que de situations associées à des émotions plus négatives. Ce n'est que pour la dernière catégorie touchant les émotions et les sensations liées à la pratique des APSA où l'on constate le contraire puisque $70,3 \%$ des incidents critiques rapportés sont jugés négatifs.

De par la place prépondérante qu'occupent les récits touchant les enseignants, les résultats obtenus rappellent à quel point les émotions des élèves peuvent être influencées par leur enseignant ainsi que par les actions qu'ils posent pour interagir avec eux, les évaluer, exposer les tâches et en réguler la réalisation, regrouper les élèves en vue de créer un environnement d'apprentissage stimulant, motivant (Watson \& Clocksin, 2013). A l'instar de Espinoza (2001), nous pensons que les élèves formulent des attentes envers leur enseignant d'EPS qui sont de nature aussi bien scolaire qu'affective et humaine.

Pour aller plus loin, les élèves qui ont l'occasion d'entretenir des liens plus personnels avec les enseignants qu'ils côtoient quotidiennement parait déterminante dans le fait de 


\section{eJRIEPS 32 avril 2014}

s'engager dans la vie de l'école, de s'intéresser aux études, de développer un sentiment d'appartenance (Richard, Dufour et Roy, 2004). Peu importe la nature des activités, la plupart des élèves apprécie l'engagement et la disponibilité des enseignants. Ces derniers sont parfois les seuls adultes capables d'offrir du soutien et d'aider les élèves qui éprouvent des difficultés d'apprentissage ou de comportement (Cyrulnik, 2004 ; Kennedy et Kennedy, 2004). L'ajustement des élèves à la vie scolaire passe beaucoup par la création de ce lien d'attachement, c'est-à-dire par la possibilité qui leur est offerte de construire un lien affectif durable avec des personnes adultes signifiantes et bienveillantes (Kennedy et Kennedy, 2004; Neufeld et Maté, 2004).

Pianta (2006) souligne également que les élèves qui perçoivent du soutien de la part de leurs enseignants montrent un plus haut degré de compétence sociale dans leurs relations avec leurs pairs de même qu'avec les adultes de l'école, sont plus engagés dans des réseaux de soutien, manifestent moins de problèmes de comportements et se montrent davantage orientés vers la réussite et vers la performance, et sont davantage susceptibles de demander de l'aide (Bear, 2010; Roehrig, Turner, Arrastia, Christensen, McElhaney \& Jakiel, 2012). À l'opposé, de mauvaises relations entre maitres et élèves ainsi qu'un mauvais climat régnant en classe ou dans l'établissement scolaire peuvent créer une perception négative de l'école et un sentiment d'aliénation menant au désengagement (Jennings \& Greenberg, 2008).

Le nombre important de récits recueillis et analysés ne permet toutefois pas de généraliser les résultats obtenus ni d'en faire la transposition à d'autres contextes sans faire montre de prudence. Ainsi, nous ne pouvons qu'appeler à une multiplication des travaux de recherche relatifs aux émotions à l'école et en EPS, de manière à saisir plus précisément les ressorts d'une notion si complexe. La question de la singularité des élèves pourrait être davantage incluse dans les travaux s'intéressant aux émotions en EPS. Lee et Solmon (2005) ont récemment réaffirmé l'importance d'étudier les variables associées aux élèves, ces derniers se présentant en cours avec des savoirs, des expériences, des valeurs et des croyances préalables qui leur sont propres. Si réellement les filles affectionnent moins l'EPS que les garçons (Lefort, 2001), la nature et l'origine des émotions en classe ne varient-elles pas selon le sexe de l'élève ? II s'agirait d'approfondir la compréhension des phénomènes affectifs en classe en tenant davantage compte de certaines variables associées aux élèves, du contexte d'enseignement-apprentissage et des variables de programmation dans le but de fournir aux enseignants des pistes d'action pour une intervention plus différenciée. 


\section{eJRIEPS 32 avril 2014}

\section{Bibliographie}

Adler, R-B. \& Towne, N. (1998). Communication et interaction, $2^{\text {ème }}$ ed., Editions Etudes vivantes.

Bear, G. G. (2010). School discipline and self-discipline. A practical guide to promoting prosocial student behavior. New York : Guilford Press.

Belzung, C. (2007). Biologie des emotions. Bruxelels : De Boeck.

Braconnier, A. (1998). Le sexe des émotions. Paris : Odile Jacob.

Brophy, J-E \& Good, T-L. (1974). Teacher-student relationships: causes and consequences. New-York, NY : Holt, Rinehart et Winston.

Brunelle, J-P., Drouin, D., Godbout, P. \& Tousignant, M. (1988). La supervision de l'intervention en activité physique. Québec: Gaétan Morin

Brunelle, J., Spallanzani, C. Lord, M. \& Petiot, B. (1983). Analyse du climat pédagogique par le biais des réactions des éducateurs physiques en situation d'enseignement. Canadian Association for Health Physical Education and Recreation Journal, 49, $15-18$ et 30 .

Canisius-Kamansi, P., Lessard, C., Riopel, M.-C., Blais, J.-G., Larose, F., Wright, A., \& Bourque, J. (2008). Les enseignants et les enseignantes au canada: contexte, profil et travail. Évolution actuelle du personnel de l'enseignement primaire et secondaire au Canada. Montréal: Chaire de recherche du Canada sur le personnel et les métiers de l'Éducation, Faculté des sciences de l'éducation, Université de Montréal.

Carron, A. V., Colman, M. M., Wheeler, J., \& Stevens, D. (2002). Cohesion and performance in sport : A meta-analysis. Journal of Sport Exercise Psychology, 24, 168-188.

Cheffers, J., \& Mancini, V. H. (1978). Teacher-student interaction. In W. G. Anderson \& G. T. Barrette (Eds.), What's going on in gym: Descriptive studies of physical education classes (pp. 39-50). Newtown, CT: Motor Skills: Theory into Practice.

Clot, Y. (1999). La fonction psychologique du travail. Paris : Presses Universitaires de France.

Creswell, J. (1994). Research design: Qualitative and quantitative approaches. London: Sage.

Csikszentmihalyi, M. (1975). Beyond boredom and anxiety. San Francisco: Jossey-Bass.

Cyrulnik, B. (2004). EPS interroge, in Revue EPS n³09.

Cyrulnik, B. (2006). De chair et d'âme. Paris : Odile Jacob. 


\section{eJRIEPS 32 avril 2014}

Damasio, A.R. (2003). Spinoza avait raison : joie et tristesse, le cerveau des émotions. Paris : Odile Jacob.

Delignières, D., \& Perez, S. (1998) Le plaisir perçu dans la pratique des APS: Elaboration d'un outil d'évaluation. Revue STAPS, 45, 7-18.

Delignières, D. (1993). Risque préférentiel, risque perçu et prise de risque. In J.P. Famose (Ed.), Cognition et performance (pp. 79-102). Paris: INSEP.

Delignières, D., \& Garsault, C. (2004). Libres propos sur l'EPS. Paris : Revue EPS.

Deslaurier, S. (2007). Plaisir perçu en E.P.S. : quels liens avec la motivation des élèves ? In Gagnaire et Lavie, Le plaisir des élèves en EPS : futilité ou nécessité ?, Coédition AEEPS-AFRAPS.

Richard, F., Dufour, M., \& Roy, G. (2004). L'encadrement des élèves au secondaire : audelà des mythes, un bilan positif. Avis au ministre de l'Éducation. Québec, QC : Conseil supérieur de l'éducation.

Dumas, D. (2009). Perceptions des comportements interpersonnels d'enseignants et d'enseignantes d'éducation physique et à la santé par des élèves du secondaire, Mémoire de Maitrise sous la direction de J-F. Desbiens, non publié.

Dumas, D., Desbiens, J-F., Spallanzani, C., \& Colin, B. (2012). L'intervention éducative en éducation physique : comment des élèves perçoivent-ils les manières d'interagir de leur enseignant? Conférence présentée le 5 juin lors du XVIlème congrès de I'AMSE-AMCE-WAER, 3 au 8 juin, centre des congrès de Reims France.

Dumouchel, P. (1999). Emotions : essai sur le corps et le social. Le plessis-Robinson : institut synthélabo.

Dweck, C. S. (1999). Self-theories: Their role in motivation, personality and development. Philadelphia: Psychology Press.

Ekman, P. (1992). Are there basic emotions ? Psychological Review, Vol 99(3), 550-553.

Espinoza, G. (2001). Rapport au savoir, rapport au maitre et affectivité : contribution à une analyse $d u$ " chemin scolaire » de l'élève. Thèse de doctorat de l'université Grenoble 2.

Famose, J. P. (2001). La motivation en EPS. Paris : Armand Colin.

Felouzis, G. (1997). L'efficacité des enseignants. Paris : PUF.

Flanagan, J-C. (1954). The critical incident. Psychological Bulletin, 51, 327-358.

Fortin, M.-F. (2010). Fondements et étapes du processus de recherche. Méthodes quantitatives et qualitatives. Montréal : Chenelière Éducation.

Fraser, B. J. (2001). Twenty thousand hours: editor's introduction. Learning Environments 


\section{eJRIEPS 32 avril 2014}

Research, 4, 1-5.

Frijda, N.H. (1996). Passions : Emotion and socially consequential behavior. In R. D. Kavanaugh, B. Zimmerberg and S. Fein (Eds.), Emotion : Interdisciplinary perspectives (pp. 1-27). Nahwah : Erlbaum.

Gagnaire, P., \& Lavie, F. (2005). Cultiver les émotions des élèves en EPS. In L. Ria (Ed), Les émotions (pp. 58-66). Paris : Editions Revue EPS.

Gagnaire, P., \& Lavie, F. (2007). Le plaisir des élèves en éducation physique et sportive : futilité ou nécessité ? Montpellier : AFRAPS.

Gagnon, J., Martel, D., Brunelle, J.P., Tousignant, M., \& Spallanzani, C. (2006). Les injustices perçues par les élèves: un message révélateur pour les enseignants. Cahiers de la recherche en éducation, 9(1), 3-20.

Galand, B., \& Philippot, P. (2005). L'école telle qu'ils la voient : validation d'une mesure des perceptions du contexte scolaire. Canadian Journal of Behavioural Science $37,2,138$.

Gordon, L. M. (1979). Enseignants efficaces : enseigner et être soi-même (Trad. Lalanne). Montréal : Edition du Jour inc.

Harkins, S. G. (1987). Social Loafing and Social Facilitation, Journal of Experimental Social Psychology, 23, 1-18.

Harkins, S. G., Latané, B., \& Williams, K. (1980). Social loafing: Allocating effort or taking it easy. Journal of Experimental Social Psychology, 16, 457-465.

Haye, G. (2011). Le plaisir. Pour l'action : ed. Revue EPS.

Jeantheau J-P., \& Murat F. (1998). Observation à l'entrée en CP des élèves du «panel 1997». Note d'information de la DPD, 40, 1-6.

Jennings, P.A. \& Greenberg, M. T. (2008). The Prosocial Classroom: Teacher Social and Emotional Competence in Relation to Student and Classroom Outcomes. Review of Educational Research, 79(1), 491-525.

Jeu, B. (1977). Le sport, l'émotion et l'espace ; essai sur la classification des sports et des rapports avec la pensée mythique ». Paris : Vigot.

Jussim, L., Yen, H., \& Aiello, J. (1995). Self-consistency, self-enhancement, and accuracy in reactions to feedback. Journal of Experimental and Social Psychology, 31, 322356.

Kennedy, J.H., \& Kennedy, C.E. (2004). Attachment theory: implications for school psychology. Psychology in the School, 41(2), 247-259.

L'Ecuyer, R. (1990). Méthodologie de l'analyse développementale du contenu. Méthode 


\section{eJRIEPS 32 avril 2014}

GPS et concept de soi. Sillery : Presses de l'Université du Québec.

Lazarus, R. S. (1984). Stress, appraisal, and coping. New York : springer.

Lazarus, R-S. (1991). "Progress on a cognitive-motivational-relational theory of emotion». American Psychologist, 46(8), 819-834.

Le Breton, D. (1998). Les passions ordinaires. Anthropologie des émotions. Paris: éditions Armand Colin.

Lee, A. M., \& Solmon, M. A. (2005). Pedagogy research through the years in RQES. The Research Quarterly for Exercise and Sport, 76(2), 108-121.

Lefort, B. (2001). Enquête sur la représentation des sciences et technologies chez les élèves de collège et de lycée de l'académie de Lille.

Lapointe, J. \& Legault, F. (1999). Version francophone du Questionnaire for Teacher Interaction en contexte québécois. Mesure et évaluation en éducation, 22(2-3), 119.

Leveau, C., Louis, E., \& Sève, C. (1999). De l'échange à la construction de la rupture, Revue EPS, 277, 43-45.

Liotard, P. (1997). L'E.P. n'est pas jouer. La maîtrise pédagogique du plaisir en éducation physique ", Corps et culture, 2, 63-87.

Martel, D., Brunelle, J., \& Spallanzani, C. (1991). La détermination du degré d'implication des participants: un indice significatif du climat d'apprentissage. STAPS, 24, 3750.

Marzouk, A., \& Tousignant, M. (1992). " Perceptions des élèves à l'égard des comportements des stagiaires dans les cours d'éducation physique ", Revue des sciences de l'éducation, 18(2), 253-267.

Maxwell, J. A. (2005). Qualitative research design. An interactive approach (2e éd). Applied Social Research Methods Series, Vol. 41. Londres: Sage.

McLeish, J. (1981). The development of modern behavioural psychology. Detselig Enterprises Ltd : Canada

Monnet, J-P. (2009). Cartaping. EPS, Cahier 12 ans et plus, 338, 3-5.

Moreau, H. (2006). Escalade : grimper en tête. Revue EPS, 322, 63-66.

Moreno, J.L. (1943). Sociometry in the classroom. Sociometry, 4.

Neufeld, G., \& Maté, G. (2004). Hold on to your kids. Toronto : Knopf Canada.

Newton, M., Duda, J.L., \& Yin, Z. (2000) Examination of the psychometric properties of the perceived motivational climate in sport questionnaire 2 in a sample of female athletes. Journal of Sport Sciences, 18, 275-290. 


\section{eJRIEPS 32 avril 2014}

Nugier, A. (2009). Histoire et grands courants de recherche sur les émotions. Revue électronique de Psychologie Sociale, 4, 8-14

Parlebas, P. (2005). Education corporelle et éducation nouvelle, conférence au congrès des CEMEA, Amiens 2005.

Pekrun, R., \& Stephens, E. J. (2012). Academic emotions. In K. R. Harris, S Graham et T. Hurden (dir.), APA educational psychology handbook, vol. 2. Individual differences and cultural and contextual factors (pp. 3-31). Washington : APA.

Pérez, S. (1999). Étude du cours d'action de professeurs expérimentés spécialistes et non spécialistes de gymnastique face à une classe de vingt-quatre, douze et cinq élèves. Thèse de doctorat STAPS non publiée, Université de Montpellier.

Perrin C. (1993). Analyse des relations entre le rapport aux APS et les conceptions de la santé, STAPS, 31, 21-30.

Pianta, R. C. (2006). Classroom management and relationships between children and teachers: implications for research and practice. In C. M. Evertson et C. S. Weinstein (dirs.), Handbook of Classroom Management: Research, Practice, and Contemporary Issues (pp. 685-709). Mahwah, N.J : Lawrence Erlbaum.

Preskill, H. (1996). The Use of Critical Incidents to Foster Reflection and Learning in HRD. Human Ressource Development Quarterly, 7(4), 335-347.

Rayou P., \& Van Zanten A. (2004). Enquête sur les nouveaux enseignants. Changeront-ils l'école ? Paris : Bayard.

Reeve, J. (2009). Understanding motivation and emotion ( $5^{e}$ éd). Danvas Ma.: John Wiley \& Son.

Ria, L. (2005). Les émotions. Collection « pour l'action». Paris : Revue EPS.

Roehrig, A.D., Turner, J.E., Arrastia, M. C., Christensen, E., McElhaney, S.,\& Jakiel, L.M. (2012). Effective Teachers and Teaching: characteristics and Practices Related to Positive Student Outcomes. In K.R. Harris, S. Graham \& T. Urdan (Eds.), APA Educational Psychology Handbook, vol. 2 (pp. 501-527). Washington DC : American Psychological Association.

Sander, D., \& Scherer, K. R. (2009). Traité de psychologie des émotions. Paris : Editions Dunod.

Sarrazin, P., Tessier, D. et Trouilloud, D. (2006). Climat motivationnel instauré par l'enseignant et implication des élèves en classe : l'état des recherches. Revue française de pédagogie, 157, 147-177.

Savoie-Zajc, L. (1994). Le discours sur l'école des jeunes identifiés à risque de 


\section{eJRIEPS 32 avril 2014}

décrochage. In L. Langevin (dir.), L'abandon scolaire : on ne nait pas décrocheur ! (pp. 79-109). Montréal, Les éditions Logiques.

Scherer, K. R., Schorr, A., \& Johnstone, T. (2001). Appraisal processes en emotion : Theory, methods, research. New-York : Oxford University Press.

Shernoff, D. J. (2012). Engagement and Positive Youth Developmenent: Creating Optimal Learning Environments. In K.R. Harris, S. Graham \& T. Urdan (Eds), APA Educational Psychology Handbook, vol. 2 (pp. 195-220). Washington DC : American Psychological Association.

Siedentop, D. (1994). Apprendre à enseigner l'éducation physique. Montréal : Gaëtan Morin éditeur.

Tardif, M., \& Lessard, C. (1999). Le travail enseignant au quotidien. Sainte-Foy : Presses de l'Université Laval.

Therme, P. (1995). L'échec scolaire, l'exclusion sociale et la pratique sportive. Paris, PUF.

Ubaldi, J-L., \& Philippon, S. (2003). Réseau d'éducation prioritaire, Quelle EPS ? Revue EPS, 299.

Visioli, J. (2009). Emotions, corps et théâtralité : contribution à une modélisation de l'expertise quotidienne de l'activité des enseignants en Education Physique et Sportive. Thèse de doctorat de l'université de Clermont-Ferrand.

Visioli, J., Petiot O., \& Ria, L. (soumis) Vers une conception sociale des émotions des enseignants?

Ward, P. (1982). Women's recreation award scheme. Brisbane, Australia: North Brisbane College of Advanced Education.

Watson, D. L., \& Clocksin, B. D. (2013). Using physical activity and sport to teach personal and social responsibility. Champaign II : Human Kinetics.

Weiner, B. (1985). An attributional theory of achievement motivation and emotion, Psychological Review, 92(4), 548-573.

Weinstein, R-S. (1985). Student mediation of classroom expectancy effects. In B. Dusek (dir.), Teacher Expectancies (pp. 329-350). Hillsdale, NJ: Erlbaum.

Wubbels, T., \& Levy, J. (1993). Do you know what you look like ? Interpersonal relationship in education. London, Washington, DC : The Falmer Press. 